\title{
Context-dependent roles of complement in cancers
}

Lubka T. Roumenina*,\#,1, Marie V. Daugan $^{*, 1}$, Florent Petitprez ${ }^{1,2}$, Catherine Sautès-Fridman ${ }^{1}$ and Wolf Herman Fridman ${ }^{\#, 1}$

1 INSERM, UMR_S 1138, Centre de Recherche des Cordeliers, Sorbonne Universités, Université de Paris, F-75006, Paris, France

2 Programme Cartes d'Identité des Tumeurs, Ligue Nationale Contre le Cancer, F-75013, Paris, France

* these authors contributed equally to the study.

\# Correspondence to:

Lubka T. Roumenina, Ph.D.

Cordeliers Research Center, INSERM UMRS 1138;

15 rue de l'Ecole de Medecine; 75006 Paris, France

Phone: 33-1-44-27-90-96/ Fax: 33-1-40-51-04-20,

e-mail: lubka.roumenina@crc.jussieu.fr

and

Wolf H. Fridman, PhD, MD.

Cordeliers Research Center, INSERM UMRS 1138;

15 rue de l'Ecole de Medecine; 75006 Paris, France

Phone: 33-1-44-27-91-02/ Fax: 33-1-40-51-04-20,

e-mail: herve.fridman@crc.jussieu.fr 


\begin{abstract}
The tumor microenvironment (TME) highly influences the growth, spreading of tumors and therefore patient's clinical outcome. In this context, complement system plays a major and complex role. It may either kill antibody-coated tumor cells or support local inflammation, hamper anti-tumor T cell responses favoring cancer spreading. Recent studies demonstrate that these opposite effects depend of the sites of its activation, the composition of the TME and the tumor cell sensitivity to complement attack. In this review, we present the implication of complement activation and its effects on cancer control and clinical outcome in different TME contexts. We also provide an overview of the publicly available transcriptomic data on the prognostic value of complement genes expression in 30 cancer types. We argue that the interplay of complement within each cancer is unique, governed by the properties of the tumor cells and the TME. This concept is of critical importance for the design of efficient therapeutic strategies.
\end{abstract}




\section{Introduction}

The interactions of malignant cells with supporting and reactive non-transformed host cells are orchestrated by the density, location and functional activity of the latter and by soluble mediators, released in the tumor microenvironment (TME) ${ }^{1}$. Frequently forgotten elements of the TME are the components of the complement system, produced by the tumor and infiltrating cells or coming from the circulation ${ }^{2}$. Complement is a key player in the innate immune defense against pathogens and in the maintenance of host homeostasis. It is composed of more than 50 plasma components produced mainly by the liver and released in the circulation or expressed on the cell membrane. They interact with each other in a cascade manner in the extracellular space ${ }^{3}$. Recent discoveries made clear that complement effectors can be generated also intracellularly and that complement proteins have noncanonical functions, independent of the cascade ${ }^{4,5}$. Cumulating evidences over the years have proven that complement proteins are present in the TME and that malignant and infiltrating cells gain capacity to produce in situ a large spectrum of these components ${ }^{6}$. Their functionality and level of expression by malignant cells or in the TME modulate the fate of the tumor. In cancer, the impact of complement is versatile, ranging from anti-tumor defense to potent tumor promotion. The data in the literature, mostly focused on animal models and in vitro studies, yield heterogeneous and sometimes contradictory conclusions. Analyses of human cancers are scarce and it is still unclear whether complement is overactivated or, on the contrary, inhibited in cancer patients. This review presents the high diversity of actions of complement components in cancer and the heterogeneity of their production and activation pathways. Using data in human cancers and in mouse models, mechanisms of tumor control and tumor promotion are discussed. We compare the expression of complement genes and their clinical impact in different cancers, suing publicly available databases and show the context-dependent impacts of complement in different cancers. Finally, we argue that the most appropriate therapeutic approaches to activate or neutralize complement are dependent on the tumor context and are difficultly transposable from one cancer to another.

\section{The complement system}

Complement is a central part of the immunity that serves as a first line of defense against pathogens and stressed host cells ${ }^{3}$. The complement system is composed of plasma proteins that react with one another to opsonize pathogens, inducing a series of inflammatory responses that concomitantly help the immune cells to fight against infections and to maintain homeostasis ${ }^{2}$. The complement cascade can be initiated, depending on the context and the location (Figure 1A).

Conventional complement activation pathways: Historically, complement was considered to be initiated by three distinct pathways - classical, lectin and alternative. Immune complexes and apoptotic cells activate the classical pathway, after recognition of the target molecules by C1q. The lectin pathway is triggered after recognition of sugar motives, foreign for a 
healthy tissue. The alternative pathway is permanently active at low grade, serving as a sentinel to attack any surface, which is not specifically protected.

Each of these pathways leads, through a sequence of conformational changes and enzymatic reactions, to the cleavage of the central component C3 into bioactive fragments C3a and $\mathrm{C} 3 \mathrm{~b}$, followed by generation of $\mathrm{C} 5 \mathrm{a}$ and the $\mathrm{C} 5 \mathrm{~b}-9$ membrane attack complex $(\mathrm{MAC})^{2,7}$. The anaphylatoxins $\mathrm{C} 3 \mathrm{a}$ and $\mathrm{C} 5 \mathrm{a}$, the opsonizing $\mathrm{C} 3$ activation fragments and the MAC are the canonical effectors of the complement system. C3a and C5a bind to their receptors $\mathrm{C} 3 \mathrm{aR}$ and $\mathrm{C} 5 \mathrm{aR}$ and play a critical role in inducing inflammation and activation of immune cells as well as endothelial, epithelial cells, fibroblasts and certain malignant cells, which express the anaphylatoxin receptors ${ }^{3}$. In support of inflammation, anaphylatoxins induce oxidative burst on macrophages, eosinophils and neutrophils. Moreover, C3a and C5a induce histamine release by basophils and mast cells to provoke vasodilatation. In a physiological context these events contribute to the acute inflammation and eradication of the pathogen. In the context of cancer, complement anaphylatoxins are constantly generated and in the majority of the studied models lead to tumor-promoting chronic inflammation ${ }^{6}$.

MAC assembly creates a transmembrane pore that causes prompt osmotic lysis of certain bacteria and metabolically inert targets (erythrocytes, lysosomes). Nucleated host cells resist lytic killing by MAC due to high expression of membrane regulators. Nevertheless, when formed, $\mathrm{C} 5 \mathrm{~b}-9$ complex can have profound effects on cell functions, leading to activation and adaptation or cell death depending on the context ${ }^{8}$. To avoid accidental healthy host tissue damage, complement is a tightly regulated cascade, constantly kept in check. However, cancer cells adapt escape mechanisms for MAC. Hsp90 protected tumor cells from complement-dependent cytotoxicity by inhibiting, together with mortalin, C5b-9 assembly and/or stability at the plasma membrane ${ }^{9}$.

Considering these effects of complement on cell activation and survival as well as on the modulation of the entire immune system, it is not surprising that the tumors are evolving to adapt to its presence and to subvert it for their benefit.

Non-canonical and intracellular complement initiation: Complement could also be activated by an unconventional, convertases-independent pathway, by enzymes cleaving $\mathrm{C} 3$ and $\mathrm{C} 5^{10-}$ ${ }^{14}$. Although generated by non-canonical mechanisms, these C3a and C5a as well as C3b and $\mathrm{C} 5 \mathrm{~b}$ are often identical in sequence with the convertase-generated anaphylatoxins. They are, therefore, canonical effectors, but generated in a non-canonical manner. This cleavage could occur in the circulation, within the tissues but also intracellularly. Interestingly, thrombin can cleave $\mathrm{C} 5$ at a different site, generating even more potent equivalent of $\mathrm{C5b}$, with higher lytic activity ${ }^{12}$.

Complement activation inside the cells has been described in T-cells and exerts homeostatic and immunological functions ${ }^{15,16}$. This intracellular complement system is referred to as a complosome ${ }^{17}$. In T-cells C3 is cleaved by cathepsin L, generating C3a and C3b ${ }^{18}$. Again in T cells (Th1), intracellular C5 activation occurs but the mechanism is still not fully defined ${ }^{19}$. 


\section{Intratumoral initiation of the complement cascade}

Despite the strong evidences of complement activation in human tumors and mouse models, only few studies addressed the pathway, by which these anaphylatoxins are generated.

Canonical mechanisms of initiation: Lung cancer is probably the best characterized model for classical pathway activation in mouse tumors. Indeed, in the seminal paper describing the pro-tumoral role of complement, a lung epithelial cell line was used (TC-1) and the complement activation was nailed down to a C4-dependent classical or lectin pathways ${ }^{20}$. Recently, we found that it was the classical pathway that was activated in this model ${ }^{21}$. In other lung cancer mouse models, the classical pathway was also activated, likely by intratumoral immunoglobulins ${ }^{22}$. Although not necessarily implicated in the complement initiation, the alternative pathway can amplify the C3 activation fragments deposits, perpetuating thus intratumoral complement activation. Little evidence is available for the implication of the lectin pathway ${ }^{23,24}$.

Non-canonical initiation: C3 and C5 can be cleaved also by complement cascadeindependent proteases, bypassing thus the initiating recognition events in cancer models ${ }^{12-}$

${ }^{14}$. C5 is cleaved by macrophages-derived urokinase in mouse models of squamous carcinogenesis, leading to $\mathrm{C} 3$-independent release of $\mathrm{C}_{5} \mathrm{a}^{13}$. Using $\mathrm{C} 5$-producing tumor cell lines, it was shown that $\mathrm{C} 5 \mathrm{a}$ can be generated by a still not identified cell membrane-bound serine protease ${ }^{14}$. Thrombin is produced in tumors and has potent pro-tumoral activity ${ }^{25}$. Therefore, it is tempting to speculate that the cleavage of $\mathrm{C} 5$ will be yet another mechanism of its pro-tumoral activity in situ.

Certain tumor cells contain intracellular pools of C3 and C5. Although currently poorly studied, we postulate that the discoveries for the intracellular cleavage of C3 and C5 made for the $T$ cells ${ }^{18,19}$ are not restricted to this population and that the intracellular generation of $\mathrm{C} 3 \mathrm{a}$ and $\mathrm{C} 5 \mathrm{a}$ may have a major role in for the biology of the tumor cells, and the nonimmune constituents of the TME, such as endothelial cell and fibroblasts.

\section{Complement effectors and immune contexture of the tumor}

Canonical functions of the complement effectors on the immune TME. The immune contexture of the tumor, which is determined by the density, composition, functional state and organization of the leukocyte infiltrate, is a key determinant of the tumor progression ${ }^{1}$. Complement receptors are expressed on the immune cells. C3a and C5a promote leucocyte attraction and impact their phenotype (Figure 2). After the discovery that C5a recruits MDSC to the tumor microenvironment and elicit them to suppress the effector $\mathrm{T}$ cells ${ }^{20}$, it was found that $\mathrm{C} 3 \mathrm{a}$ and/or C5a exert a profoundly influence on the TME by inducing a series of context-dependent functions, including: 1) recruitment of tumor-promoting macrophages and $\mathrm{CCL}_{2}$ production ${ }^{26}, 2$ ) decrease of $\mathrm{CD} 4+$ T-cells and neutrophils recruitment ${ }^{27}, 3$ ) decrease of NK cells recruitment ${ }^{28}$, 4) stimulation of a pro-tumoral phenotype for CD4+ T- 
cells $\left.{ }^{22}, 5\right)$ inhibition of IL10 expression in intratumoral CD8+ T-cells $\left.{ }^{29}, 6\right)$ stimulation of the protumorigenic properties of mast cells and macrophages, including suppression of CD8+ T cell cytotoxicity ${ }^{13}$, 7) promotion of pro-tumoral NETosis by neutrophils ${ }^{30}$, etc.

The level of intratumoral C5a may be a key determinant for the composition of the immune TME. Mouse lymphoma producing low level of C5a grow slower in mice and have increased IFN- $\gamma$-producing T-cells in spleen and tumor-draining lymph nodes ${ }^{31}$. Conversely, tumorbearing mice with high C5a-producing cells had accelerated tumor progression with more Gr-1+CD11b+ myeloid cells in spleen and overall decreased T-cells in tumor, tumor-draining lymph nodes, and spleen.

The C3 activation fragments are potent effectors, modulating the immune response ${ }^{3}$. Chemo- as well as immuno-therapeutical approaches induce apoptosis in tumor cells. Opsonization of apoptotic tumor cells with iC $3 \mathrm{~b}$ prevents the maturation of the dendritic cells via interaction with CR3 and contribute to the induction of antigen-specific silencing and tolerance ${ }^{32}$. Moreover, iC3b-CR3 interaction results in dysregulation of NK-dependent tumor surveillance ${ }^{33}$.

Even though the majority of the experimental models agree on the pro-tumoral role of C3a and especially $\mathrm{C} 5 \mathrm{a}$, the mechanisms described above are context-specific. Rarely the same mode of action of the anaphylatoxins is found in different cancer models. This could reflect the differences in the composition of the immune microenvironment of every cancer. Indeed, in mice, as in humans, the immune infiltration is largely controlled by the properties of the tumor cells themselves ${ }^{1,34}$. Whereas a large body of data concerning the impact of complement in different murine models and in vitro are available, those in human cancers remain scarce.

Non-canonical impact of complement components on immune cells. By their non-canonical functions, complement components modulate the fundamental processes of immune cells, including immune cells proliferation, migration, metabolism, and even transcriptional activity $^{35-37}$. Studies on human $\mathrm{T}$ cells show unconventional intracellular C3 cleavage by cathepsin $\mathrm{L}^{18}$. This "tonic" intracellular C3a is required for homeostatic T cell survival. At least in part this $\mathrm{C} 3$ was internalized as $\mathrm{C} 3(\mathrm{H} 2 \mathrm{O})$ from the extracellular milieu ${ }^{38}$. The cleavage of C3 by cathepsin L is species-specific and does not operate in mice ${ }^{18}$. This should be taken into account if this mechanism is studied in animal models of cancer. Again in human T cells (Th1), intracellular C5 activation and stimulation of intracellular C5aR1 results in the assembly of the NLRP3 inflammasome, needed for the optimal production of IFN- $\gamma^{19}$. Moreover, novel functions of intracellular C3 emerge, such as its implication in immune cells gene transcription ${ }^{37}$ or regulation of autophagy ${ }^{39}$. C1q was shown to modulate CD8+ T cell metabolism in the context of autoimmunity and viral infections ${ }^{36}$. All these processes have not been studied in the context of cancer yet. Nevertheless, it is tempting to speculate that the non-canonical functions of complement will shape the immune TME and will play a key role in anti-tumor immunity. 


\section{Neoangiogenesis and complement}

Neovascularization is critical for the oxygen and nutrients supply to the tumor. Complement contributes to this process via its canonical effectors $\mathrm{C} 3 \mathrm{a}$ and $\mathrm{C} 5 \mathrm{a}$ as well as by noncanonical, cascade-independent effects of the individual components (Figure $3 \mathrm{~A}$ )

Mode of action of the canonical effectors: It has been known for long that C5a promotes migration and tube formation of endothelial cells in vitro ${ }^{40,41}$. In addition, C3-/- and C5aR-/endothelial cell have impaired angiogenesis capacity ${ }^{42}$. Nevertheless, the impact of C3a and C5a in mouse tumors seems to be model-dependent. C3-/-, C3aR-/- or C5aR-/- showed either impaired tumor angiogenesis ${ }^{13,42}$, increased blood vessel permeability without effect on microvascular density ${ }^{43}$ or no impact $^{41}$. The context-dependent impact of the anaphylatoxines on neoangiogenesis requires further investigation.

Mode of action of the non-canonical effectors: Recent evidences point towards a major role of $\mathrm{C} 1 \mathrm{q}$ in cancer neoangiogenesis via non-canonical, cascade-independent mechanism. A fraction of tumor vessels endothelial cells produce $\mathrm{C} 1 \mathrm{q}$ in mouse models and in human tumors $^{21,44}$. The microvascular density was either decreased or the vascular network was disorganized in tumors, growing in C1q-/- mice ${ }^{21,44}$. This could be explained by alteration of the C1q-mediated expression of VEGFs and VEGFRs, as shown in tumor models ${ }^{21}$ and in studies of pregnancy complications of these mice ${ }^{45}$. The pro-angiogenic effect of $\mathrm{C} 1 \mathrm{q}$ is again context-dependent, since neoangiogenesis was enhanced in neuT-C1q-/- breast cancer model $^{46}$.

Although mouse models provide insights for the role of complement cascade and its individual components in tumor neoangiogenesis, most of the data are generated with subcutaneously implanted tumor cell lines. Endothelial cells from different organs have unique properties, including different spectrum of complement proteins expression ${ }^{47,48}$, which may differentially impact the neoangiogenesis in the cancer types. Therefore, further studies are needed to determine the relative impact of complement in neoangiogenesis of human tumors.

\section{Direct impact of complement effectors on tumor cell biology}

Impact of the canonical effectors: In addition to promoting inflammation, C3a and C5a could affect the fundamental processes of the tumor cells, such as survival, proliferation, migration, stemness, etc (Figure 3B,C). Anaphylatoxin receptors are expressed on certain cancer cells ${ }^{6}$. Multiple reports show that these cells express also $\mathrm{C} 3$ and/or $\mathrm{C} 5$ and generate $\mathrm{C} 3 \mathrm{a}$ and $\mathrm{C5a}$, acting in an autocrine manner. The impact of the signaling is related to stimulation of proliferation ${ }^{49,50}$, multipotent state of glioblastoma glioma stem-like cells ${ }^{51}$, the epithelial to mesenchymal transition ${ }^{52,53}$, invasiveness and morphology alteration ${ }^{54}$, stemness etc. For example C3a enhanced cell proliferation, migration and stemness in cutaneous squamous cell carcinoma and this activity was correlated with activation of the Wnt and $\beta$-catenin pathway ${ }^{55}$. If the cascade proceeds to the terminal MAC formation, it was 
shown that the sublytic levels of C5b-9 mediate signaling, promoting cancer cell cycle progression ${ }^{56}$. Cancer cells-derived $\mathrm{C} 3 \mathrm{a}$ also adapts the cerebrospinal fluid for leptomeningeal metastasis by activation of $\mathrm{C} 3 \mathrm{aR}$ on the choroid plexus epithelium, disrupting thus the blood-cerebrospinal fluid barrier ${ }^{57}$. The role of complement for cancer metastases is reviewed elsewhere ${ }^{58}$.

Complement cascade may lead to tumor cell killing if sufficiently strongly activated by host anti-tumoral IgM or IgG or by therapeutic antibodies and if abundant MACs are inserted into the cell membrane. In the context of cancer, little evidence suggests that complement can proceed to cell-killing MAC without treatment with targeted therapeutic (like tumor-cell targeting monoclonal antibodies ${ }^{59}$ ). This escape from complement killing is in part linked to a high expression of complement regulators at tumor cell surface.

Non-canonical functions of the complement proteins: Many complement components, such as $\mathrm{C} 1 \mathrm{q}, \mathrm{C} 1 \mathrm{~s}, \mathrm{C} 3$, properdin, $\mathrm{FH}, \mathrm{Fl}$, etc have non-canonical, extracellular and intracellular functions, modulating the fundamental processes of the tumor cell in selected models, promoting proliferation and tumor progression when tested in animal models ${ }^{44,60-62}$. In embryonic development and in cancer models, intracellular C3 impacts epithelial to mesenchymal transition ${ }^{52}$. Properdin, which is the only positive regulator of the cascade, has here also opposite non-canonical functions, compared to the majority of the other tested

complement proteins. It suppresses breast cancer cell growth by control of transcription ${ }^{63}$. C1q could exert anti-tumoral effects by activation of tumor suppressor WWOX to induce apoptosis in prostate and breast cancer cells ${ }^{46,64}$. WWOX is only weakly expressed in the majority of the tumor types, suggesting that this effect of C1q will be context-dependent, relevant particularly of the hormone-regulated tissues (breast, ovary, prostate, etc).

\section{Complement components and receptors in human tumors}

The potential roles of complement as a part of the interplay between malignant cells and the TME in human cancer begins to unveiled. Although quite dispersed, literature accumulates to show that tumors develop in a complement rich milieu. Many of the cells, present in the TME, produce complement components and/or bear complement receptors and complement regulators ${ }^{65}$, suggesting a potential in situ activation of the complement pathways (Figure 1B). In a physiological context the complement components produced by the immune cells regulate the fundamental processes of the cells and help to fight infection. Nevertheless, in the context of the tumor, the malignant cells may produce various complement components, resulting in a disturbed complement milieu, impacting local complement activation. From the analysis of the literature, two striking observations can be made. The first one is the presence of complement receptors, particularly C3aR and C5aR on most of the cell types in the TME, suggesting that the activity and functions $T$ and $B$ lymphocytes, neutrophils, macrophages, MDSCs, DCs, endothelial cells and fibroblasts can be modulated by the activation fragments of $\mathrm{C} 3$ and $\mathrm{C} 5$. The second is the presence at high levels of complement regulators, capable to inhibit complement activation, particularly the 
terminal pathway, on malignant cells. This is an underscored mechanism of escape of the tumor cells from the attack of complement, which can add up to the immune escape mechanisms that could guide cancer Immunotherapy ${ }^{66}, 67$. This may explain also why intratumoral C5b-9 staining has not been frequently reported ${ }^{68}$. Unfortunately, only very limited number of studies address the expression and activation of complement in large cohorts of patients with different cancers. This problem can be at least in part resolved now, thanks to the data mining and bioinformatics analyses of The Cancer Genome Atlas (TCGA) database and the pathology atlas of the human cancer transcriptome (protein atlas) ${ }^{69,70}$.

\section{Expression of the complement genes in human cancers}

To draw an overall picture of the impact of complement in different cancers, we compared the gene expression levels and the prognostic impact of the main complement components in 29 tumor types, using publicly available datasets ${ }^{69,} 70$. Figure 4 shows a non-supervised hierarchical clustering of the expression of 50 complement-related genes in solid tumors, encompassing 30 cancers. Several conclusions can be drawn from this analysis. Overall, there is a strong heterogeneity in expression among genes but, surprisingly, not that much between cancer types. The gene encoding C3, the pivotal complement component, is expressed in all cancer types together with genes of the components of the classical pathway (C1QA, C1QB, C1QC, C1R, C1S, C4A and C2). In contrast, genes for the components of the lectin pathway are poorly expressed in all tumor types (MBL2, MASP2, FCN2) or heterogeneous with poor expression in the majority of the cancers (MASP1, FCN1, FCN3), arguing against a major implication of this pathway in in situ activation of the complement cascade. As for the alternative pathway, CFB and CFD are heterogeneously expressed with a particular low expression in kidney chromophobe (KICH), uveal melanoma (UVM), prostate adenocarcinoma (PRAD). The remaining tumors exhibit a higher expression of CFB and CFD. This, together with the high local expression of $C 3$, suggests that complement could be activated via the classical or the alternative pathway.

A striking feature is the very low expression of $C 6, C 8 A, C 8 B$ and $C 9$ genes (with the exception of cholangiocarcinoma ( $\mathrm{CHOL}$, bile duct cancer), which suggests that terminal pathway is unlikely to be activated via in situ produced components. Moreover, the genes encoding complement regulators acting at the level of C1 (SERPING1, C1 inhibitor) and at the level of the $\mathrm{C} 3$ convertases, (CFH, CFI, CD46 and CD55) are highly expressed in most cancers. Moreover, the terminal pathway regulator CD59 is among the highest expressed complement genes in all studied tumors, suggesting efficient protection of the malignant cells from complement-mediated killing. This pattern of gene expression is perfectly in line with the examples from the literature, demonstrating high expression of these regulators in different types of cancer ${ }^{68,71-76}$. Complement-mediated cytotoxicity may act as a selective pressure for tumor overexpression of complement regulators. Indeed, hypoxic tumor cells are resistant to complement-mediated cytotoxicity due, in part, to hypoxia-induced expression of complement regulator CD55 in colorectal cancer ${ }^{77}$. This is again contextdependent, since in an NSCLC model, on the contrary, hypoxia decreased the regulators expression and increased susceptibility of the tumor cells to complement attack in vitro ${ }^{78}$. 
The low levels of terminal pathway genes expression together with the high expression of complement regulators reinforce the hypothesis that malignant cells evolve and adapt to avoid potentially killing MAC formation. Instead, intratumoral complement activation can be sustained via locally expressed classical and alternative pathway components, generating thus the largely pro-tumoral anaphylatoxins C3a and C5a.

\section{The prognostic impact of the complement expression in cancer patients}

Further, we evaluated the impact of the expression of genes encoding components of the classical and alternative pathways on overall survival of patients with different malignancies, utilizing data available in the TCGA (Figure 5). Four groups of cancers could be defined. The first contains tumor types, for which the overexpression of the genes of the components of the classical and alternative pathway are associated with good prognosis. This group of cancers with "protective complement" involves prostate adenocarcinoma (PRAD), mesothelioma (MESO), sarcoma (SARC) and skin cutaneous melanoma (SKCM). A second group in which C3 expression correlates with longer overall survival (OS) comprises kidney chromophobe (KICH), adrenocortical carcinoma (ACC) and thyroid cancer (THCA), although significance is not reached in the latter. The third group contains cancers in which high expression of classical and alternative pathway genes correlates with poor prognosis. This group of "aggressive complement" tumors includes uveal melanoma (UVM), low grade glioma (LGG), glioblastoma (GBM), kidney clear cell renal cell cancer (KIRC), lung squamous cell carcinoma (LUSC) as most significantly impacted types. It includes also digestive tract cancers such as: rectum adenocarcinoma (READ), colon adenocarcinoma (COAD) and stomach adenocarcinoma (STAD), as well as uterine cancers, like: uterine corpus endometrial carcinoma (UCEC) and uterine carcinosarcoma (UCS), for which significance was not reached for most of the genes. The fourth group encompasses a large number of tumor types in which the gene expression analysis did not reveal any robust clinical impact, named therefore of "uncertain significance". It comprises cholangiocarcinoma (CHOL), cervical squamous cell carcinoma and endocervical adenocarcinoma (CESC), breast invasive carcinoma (BRCA), pancreatic adenocarcinoma (PAAD), lung adenocarcinoma (LUAD), urothelial bladder carcinoma (BLCA), head-neck squamous cell carcinoma (HNSC), ovarian cancer (OV), lymphoid neoplasm diffuse large B-cell lymphoma (DLBC) and thymoma (THYM).

Cancers with "protective complement": It is striking that within this group, the density of B cells and B cell transcriptomic signatures were associated with longer survival and response to immunotherapy with immune check point blockers in melanoma (Jennifer Wargo, unpublished results) and in soft tissue sarcoma (WHF, unpublished results). Therefore, it is tempting to speculate that in these cases complement is involved in the beneficial effect of $B$ lymphocytes. Indeed, early studies from the 60 s have already noticed a potential link between the presence of anti-sarcoma antibodies and anti-tumoral effect of complement in mice $^{79}$ and complement-fixing antibodies were detected in sera from sarcoma patients ${ }^{80}$. High expression of $\mathrm{C} 5$, produced mainly by the tumoral cells, correlated with better event- 
free and overall survival Ewing's sarcoma ${ }^{81}$ (not included in the TCGA dataset). In these cases, C5aR was mainly detected on tumor cells in situ.

Despite the abundant set of data about complement in mice grafted with melanoma cell lines (yielding often contradictory conclusions ${ }^{27-29,}{ }^{44}$ ), little is known about the complement activation in human melanoma and its association with clinical parameters in patients' cohorts.

Very limited data is available for complement and prostate cancer. Sublytic complement C5b-9 protects prostate cancer cells from tumour necrosis factor- $\alpha$-induced cell death ${ }^{82}$. Interestingly, proteolysis of $\mathrm{iC} 3 \mathrm{~b}$ and $\mathrm{C} 5$ by the serine protease prostate-specific antigen in prostatic fluid was detected, inhibiting the terminal pathway ${ }^{83}$.

The mesothelioma (MESO) presents an interesting case. The analysis presented above includes only 82 patients with heart, mediastinum and pleural MESO and shows overall tendency of good prognosis associated with classical and alternative complement genes. Experimental evidences are available only for pleural MESO. Evaluation of C4d and C1q staining in a malignant pleural MESO cohort revealed absence of C1q staining in the majority of the tumors (only few positive infiltrating immune cells) and absence of $\mathrm{C} 4 \mathrm{~d}$ deposits on malignant cells ${ }^{84}$. Membranous $\mathrm{C} 4 \mathrm{~d}$ deposits were found only in tertiary lymphoid structures and this staining was associated with bad prognosis. Patients with low C4d plasmatic levels at diagnosis had a significantly better overall survival. In another cohort, C1q staining of tumor and infiltrating myeloid cells was strong ${ }^{85}$. In this context C1q was shown to bind to hyaluronic acid and promote cell adhesion and proliferation in complement cascadeindependent manner.

Further studies are needed to clarify the potential link between complement and cancer progression in the group of "protective complement" in order to provide experimental validation whether the concerted expression of these proteins occurs indeed in situ and whether complement is indeed activated. A staining of large cohorts of patients for $\mathrm{C} 1 \mathrm{q}$, C4d, C3d and C5b-9 is necessary to determine the real impact of complement for cancer progression and prognosis in these groups of patients.

Cancers with "protective C3": In thyroid cancer (THCA) cellular deposits of IgG and complement factors $\mathrm{C} 3 \mathrm{~d}, \mathrm{C} 4 \mathrm{~d}$, and $\mathrm{C} 5$ were shown in up to $80 \%$ of the cases, but data for prognostic impact was not reported ${ }^{86}$. Since these cancers are of particularly good prognosis, this may go in line with the lack of prognostic impact of complement in this cancer. No data from the literature was available to verify in situ the impact of complement in $\mathrm{KICH}$ and ACC, for which the high expression of $\mathrm{C} 3$ gene is associated with particularly good prognosis. In these cancers the protective role of $\mathrm{C} 3$ could potentially be driven by non-canonical functions.

Cancers with "aggressive complement": Our analysis reveals a particularly strong negative prognostic impact of the complement genes in uveal melanoma (UVM). A high expression 
level of complement regulators has been reported for this cancer ${ }^{87}$ but no data are available for the status of complement activation in situ. Further studies are warren to test whether indeed in UVM complement expression/deposits may impact prognosis and to evaluate whether patients with this cancer may benefit from complement blocking therapy.

We suggest to classify gliomas as cancers with "aggressive complement". The complement components C1QA, C1S, C2 and C7 were found upregulated in high risk relative to low risk glioma (GBM and LGG) patients ${ }^{88}$. The activation of $\mathrm{B}$ cells around high-risk gliomas is also likely, as indicated by the enrichment of a B cells related gene set, over-expressed in high risk compared to low risk glioma. Other studies also suggest that complement is activated in GBM, but the deleterious impact remains to be proven by in situ analyses ${ }^{51,89}$.

To date the best examples of "aggressive complement" tumors, for which genes expression and bioinformatics analyses corroborate with assessment at protein level in situ are KIRC and non-small cell lung cancer (NSCLC). In both cases all components for classical pathway activation could be produced intratumorally and classical pathway activation, detected by presence of C1q and C4d-positive deposits was associated with poor prognosis ${ }^{21,90}$.

The classical pathway requires a trigger. C1q can bind over 100 different targets, but the major ones are the IgG and IgM-containing immune complexes ${ }^{2}$. Indeed, IgM and IgG antibodies have been detected in several tumor types such as in $\mathrm{NSCLC}^{91}$, ovarian ${ }^{92,93}, \mathrm{KIRC}^{21}$ and breast ${ }^{94}$ cancers. These antibodies may come from the circulation or be produced at the tumor site ${ }^{91,93}$ potentially initiating the complement cascade. Indeed, in $\mathrm{KIRC}^{21}$ and in $\mathrm{NSCLC}^{90}, \mathrm{C} 1 \mathrm{q}$-mediated classical pathway activation was detected, leading to C4d deposits. In both cases high level of intratumoral C4d staining was associated with poor prognosis ${ }^{21,90}$. Also, C4d in plasma was increased in NSCLC patients, associated with poor prognosis ${ }^{90,95}$. Data in the literature do not present separately the two subtypes of NSCLC: lung squamous cell (LUSC) and lung adenocarcinoma (LUAD). Although LUSC falls in the "aggressive complement" group, for LUAD the significance of complement is uncertain.

The assessment of complement genes expression in different tumors strongly suggest that when complement is activated in situ, this happens thanks to locally produced complement proteins. Data are lacking for the majority of the cancer types, but within the group of tumors with "aggressive complement", we evaluated the complement status at protein level in KIRC patients ${ }^{21}$. We found that there is an in situ orchestrated production of C1q by tumor-associated macrophages (TAMs) and $\mathrm{C} 1 \mathrm{r}, \mathrm{C} 1 \mathrm{~s}, \mathrm{C} 4$ and $\mathrm{C} 3$ by tumor cells, concomitant with IgG deposits. This allowed C1 complex assembly and complement activation. Interestingly, what conferred poor prognosis in KIRC patients was the presence of intratumoral $\mathrm{C} 1 \mathrm{q}$ producing TAM, as well as the concomitant local production and deposition of $\mathrm{C} 4$ activation fragments on the tumor cells. A surprising finding was that the local production of $\mathrm{C} 3$ by tumor cells conferred poor prognosis, while C3d-positive deposits were not associated with prognostic impact. This, together with the clear negative impact of C3 in different mouse models, it is important to evaluate the spectrum of deposits of C3 activation fragments in human tumors. Indeed, the used antibodies did not allow to determine the exact nature of the fragments, the anti-C3d recognizing $\mathrm{C} 3, \mathrm{C} 3 \mathrm{~b}$, iC3b as well as the C3dg and C3d fragments, each having different function. In another cohort, though, 
the C5a-positive staining (marking indistinguishably the presence of the anaphylatoxin $\mathrm{C} 5 \mathrm{a}$ and the intact $\mathrm{C} 5$ ) and the presence of $\mathrm{C} 5 \mathrm{aR}+$ staining were correlated with poor prognosis ${ }^{96,}$ 97. The malignant cells in KIRC, therefore, hijack macrophage-produced C1q to complement the remaining components made by the cancer cell, to promote tumor growth. The proposed mechanism of action in tumors with "aggressive complement" is depicted in Figure 6, based mainly on the data from KIRC and NSCLC, as well as on mouse and in vitro data. Further studies are needed to evaluate to what extend this mode of action of complement is valid for other cancers.

Cancers with "complement of uncertain significance": This group encompasses a large number of cancers, for which either sporadic significance for the prognostic impact was detected or no significance at all, using the median cutoff. Interestingly, in pancreatic adenocarcinoma (PAAD) tumor cells release exosomes, which harbor $B$ cell targets and bind anti-tumoral IgG to exert decoy function against potential complement-mediated cytotoxicity $^{98}$. This could explain why, despite the high expression of classical and alternative pathway genes and proteins ${ }^{99}$ in PAAD, they do not seem to be associated with prognosis. Complement may not be efficiently activated in situ, when IgGs are subverted from the tumor cells and targeted to exosomes.

\section{Cascade-independent impact of complement in human cancers}

As evidenced in in vitro and mouse models, most of the complement proteins have functions outside the cascade. They can operate alone and/or in parallel with the cascade in any of the tumor groups defined here. An indication for such phenomena could be if one gene or expressed/deposited protein is associated with good or poor prognosis, while the remaining members of the same pathway are not. Another indication could be if the presence of the protein within the cell is associated with a prognostic impact, while its deposits are not.

This could be illustrated with the case of C1q-producing TAM in KIRC. We found that they were a robust marker for poor prognosis in three independent cohorts. Since C1q was produced by $\mathrm{M} 2$-like subtype of TAM, the question arose whether this negative impact illustrates the well-known pro-tumoral impact of the immunosuppressive and proangiogenic $\mathrm{M} 2^{100-102}, \mathrm{C} 1 \mathrm{q}$ being an additional biomarker for this population or whether $\mathrm{C} 1 \mathrm{q}$ plays a role in the balance between TAM phenotypes, influencing their mode of action. Interestingly, a recent in-depth immune profiling revealed that TAMs in KIRC (but likely in other cancers as well) represent a heterogeneous cell population with a subset called M-5 being associated with T-cell exhaustion ${ }^{103}$. TAMs of the M-5 subset express higher levels of $\mathrm{C} 1 \mathrm{q}$ genes as well as $\mathrm{C} 1 \mathrm{q}$ receptors and $\mathrm{C} 3 \mathrm{aR}$, making them responsive to $\mathrm{C} 1 \mathrm{q}$ and $\mathrm{C} 3 \mathrm{a}^{21}$. In addition, they overexpress PD-L2. Both $\mathrm{M}-5$ and $\mathrm{C}_{1} \mathrm{q}^{+} \mathrm{TAM}$ were associated with T-cell exhaustion. Since immunosuppressive action of C1q was already described for T-cells ${ }^{36,104}$, 105 , it is tempting to speculate that M-5 macrophages exert their immunosuppressive activity at least in part via $\mathrm{C} 1 \mathrm{q}^{21}$. 


\section{Warnings on the use of transcriptomic data for complement genes to predict patients' outcome}

Gene expression analyses may inform on the potential production level of their corresponding protein, but in the complement cascade the generation of the effectors is a matter of activation and cleavage. Although transcriptomic analyses are useful to pinpoint associations between complement components and clinical outcome, only in situ analyses of the complement proteins, their activation fragments deposits ( $C 4 d, C 3 b, i C 3 b, C 3 d)$, the anaphylatoxins ( $\mathrm{C} 3 \mathrm{a}, \mathrm{C} 5 \mathrm{a})$, their receptors ( $\mathrm{C} 3 \mathrm{aR}, \mathrm{C5aR} 1, \mathrm{C5aR2})$ and their regulators will allow to understand how complement modulates tumor cells and the TME, resulting in control or development of cancers.

A clear example for such discrepancy is the modest prognostic impact of C3AR and C5AR1 genes, which reach statistical significance at median cutoff only for 3 and 5 cancers respectively. Nevertheless, C5aR seems to be the key effector of the deleterious impact of

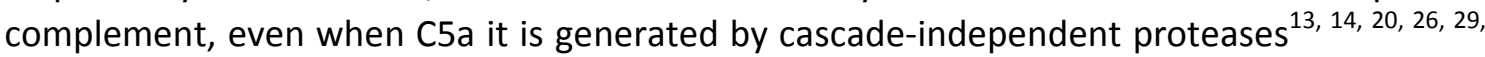

${ }^{106}$. In addition, a clear negative prognostic impact was detected for $\mathrm{C} 1 \mathrm{~s}$ in bladder urothelial carcinoma (BLCA) at protein level ${ }^{107}$. Detailed gene expression analyses of the TCGA cohort revealed that $C 1 S$ as the most significantly upregulated gene related to advanced disease both in urothelial carcinoma of upper tract and in urinary bladder cancer. It correlated with a panel of disease markers, but the gene expression failed to reach significance in terms of prognostic impact. This case illustrates the importance to perform staining at protein level to determine the prognostic impact of a given protein.

Another hurdle is that different activation fragments of the complement proteins have different biological functions. Therefore, the exact deposited activation fragment has to be distinguished in situ. Well characterized and validated antibodies with known fragment specificity should be used. Detection of complement and its activation fragments is performed in routine pathology laboratories and the difficulties to work with paraffin embedded tissues are well documented ${ }^{108}$. Nevertheless, nowadays reliable protocols exist for staining of complement components and activation fragments in paraffin-embedded tumor tissue ${ }^{21}$, with antibodies, validated by competition tests with intact proteins or fragments. With the current state of the image analyses, distinction between intracellular production and deposits in automated algorithms is tricky. This requires experienced observers to stratify the patients. Moreover, proteomic analyses allow spatially resolved profiling of the proteins within different tumor regions. The complement exploration in cancer will benefit from the advent of this technology, which will allow to better evaluate the complement production and deposits in situ.

A further challenge, when a complement protein is produced both by malignant and by infiltrating cells, is to ascribe the pro- or anti-tumoral effect to this complement protein and its canonical and/or non-canonical functions, or to the presence of the cell itself, as it was the case for $\mathrm{C} 1 \mathrm{q}+\mathrm{TAM}^{21}$. 
Indeed, our prognostic data need to be interpreted within the limits of using median gene expression as a cutoff to stratify patients. Even if the median cut off did not reveal significant prognostic impact for complement genes in the "uncertain significance" group of patients, use of other cut-offs may have unveiled significant correlations.

\section{Therapeutic perspectives.}

An analysis on a large panel of cancers (Figure 4) revealed simultaneous intratumoral expression of genes coding for proteins involved in complement activation (C1q, C1s, C1r, $\mathrm{C} 3$ ) or sensing activation products ( $\mathrm{C} 3 \mathrm{aR}$ and $\mathrm{C} 5 \mathrm{aR}$ ) as well as in complement regulation (C1Inh, FH, FI, CD59). Taken together, the local production and increased activation of complement in the tumor microenvironment is associated with dampening of the anti-tumor immune responses and promotion of cancer development in a large subset of cancers. Despite the heterogeneity of the data in the literature, it can be concluded that in the majority of the tumors the anaphylatoxin receptors C3aR and C5aR are novel class of immune checkpoints that could be targeted for tumor immunotherapy. Indeed, C5aR blockade showed decreased tumor growth in variety of mouse models alone ${ }^{6}$ or in combination with a checkpoint inhibitors (anti-PD1, anti-PDL1) ${ }^{29,109,110}$. Moreover, a phase 1 clinical trial for administration of anti-C5aR monoclonal antibody (IPH5401) in combination with anti-PDL1 Durvalumab in patients with advanced solid tumors is now recruiting (STELLAR-001, NCT03665129). C5aR blockade could improve also the efficacy of chemotherapy. Indeed, C5aR targeting with PMX-53 improved efficacy of paclitaxel chemotherapy, by promoting antitumoral $T$ cell response ${ }^{13}$. Many more complementtargeting molecules are in the pipeline for variety of disease indications ${ }^{111}$ and can be adapted for cancer therapy, acting at different steps of the cascade ${ }^{112}$. Nevertheless, the therapeutic combinations have to take into account the large variety of functions of complement proteins, such as in the context of radiotherapy, where C3a and C5a are crucial to the anti-tumor immune response ${ }^{113}$.

Cancer vaccines are a promising approach to stimulate the immune system to efficiently recognize and kill tumor cells. Endothelial quiescence prevents tumor-specific T-cells homing. This endothelial quiescence was reversed by cytokine-mediated activation of the tumor vasculature followed by upregulation of C3 and local generation of C5a in the TC-1 mouse model of tumor vaccination ${ }^{114}$. The $\mathrm{C} 5 \mathrm{a}$-dependent upregulation of endothelial adhesion molecules, resulted in efficient T-cell extravasation, infiltration into the tumor and malignant cells killing. These results highlight once again the context-dependent action of complement. In the same model complement activation is pro-tumoral ${ }^{20,21}$, but turns antitumoral in case a robust antitumor immune response ${ }^{114}$. Indeed, these data suggest that when effector $T$ cells are present, complement facilitates tumor rejection, whereas it may promote inflammation and tumorigenesis when other immune cell types predominate over antitumor $\mathrm{T}_{\text {cells }}{ }^{114}$.

Big efforts are now focused to design anti-cancer monoclonal antibodies with enhanced complement mediated cytotoxicity in order to kill the tumor cells to which they are directed. 
The recent discovery of the potentiation of the C1q binding and complement activation by IgG hexamerization brought a new trend for generation of a new generation of therapeutic antibodies $^{115-117}$. If given in the right context, they could indeed confer benefit in the eradication of the tumor. Although limited, the data from the literature suggest that the impact of malignant cells-binding IgG on tumor growth is context dependent. In an immunostimulatory milieu, such antibodies can induce powerful anti-tumor immunity that can potentially be harnessed for the treatment of patients with cancer. Potential benefit to harm ratio has to be evaluated for each subset of patients even within one tumor type to avoid potential enhancement of the pro-tumoral impact of complement. Intervention strategies, recently summarized by Fishelson and Kirschfink ${ }^{118}$ have to be established to overcome the resistance of the tumor cells to complement mediated killing, which will improve the efficacy of these therapeutic antibodies.

In order to design efficient complement targeted therapeutics for cancer we have to better understand the mechanism by which these complement proteins contribute to tumor development. This will allow to tip the fine-tuned balance of the complement reaction and to decide whether to block the activation, prevent the regulation and/or act on the functions of these proteins outside complement system.

\section{Acknowledgement}

This work was supported by a grant from Pierre Fabre Research Institute (to WHF and LR), grants from Association pour la Recherche sur le Cancer (ARC), the Cartes d'Identité des Tumeurs (CIT) Program from the Ligue Nationale Contre le Cancer (RS19/75-111) and Cancer Research for Personalized Medicine (CARPEM) to LTR; Institut du Cancer (INCa) HTE Plan Cancer (C1608DS) to CSF, PRTK G26 NIVOREN and BioniKK (to CSF) programs. This work was also supported by INSERM, University of Paris, Sorbonne University, CARPEM T8, and the Labex Immuno-Oncology Excellence Program. MVD received a PhD fellowship from ARC, and FP a doctoral fellowship from CARPEM.

\section{References}

1. Fridman, W.H., Zitvogel, L., Sautes-Fridman, C. \& Kroemer, G. The immune contexture in cancer prognosis and treatment. Nat Rev Clin Oncol 14, 717-734 (2017).

2. Merle, N.S., Church, S.E., Fremeaux-Bacchi, V. \& Roumenina, L.T. Complement System Part I Molecular Mechanisms of Activation and Regulation. Front Immunol 6, 262 (2015).

3. Merle, N.S., Noe, R., Halbwachs-Mecarelli, L., Fremeaux-Bacchi, V. \& Roumenina, L.T. Complement System Part II: Role in Immunity. Front Immunol 6, 257 (2015).

4. Kemper, C. \& Kohl, J. Back to the future - non-canonical functions of complement. Semin Immunol 37, 1-3 (2018).

5. Kolev, M., Le Friec, G. \& Kemper, C. Complement--tapping into new sites and effector systems. Nat Rev Immunol 14, 811-20 (2014).

6. Reis, E.S., Mastellos, D.C., Ricklin, D., Mantovani, A. \& Lambris, J.D. Complement in cancer: untangling an intricate relationship. Nat Rev Immunol 18, 5-18 (2018). 
7. Gros, P., Milder, F.J. \& Janssen, B.J. Complement driven by conformational changes. Nat Rev Immunol 8, 48-58 (2008).

8. Morgan, B.P., Walters, D., Serna, M. \& Bubeck, D. Terminal complexes of the complement system: new structural insights and their relevance to function. Immunol Rev 274, 141-151 (2016).

9. Rozenberg, P., Ziporen, L., Gancz, D., Saar-Ray, M. \& Fishelson, Z. Cooperation between Hsp90 and mortalin/GRP75 in resistance to cell death induced by complement C5b-9. Cell Death Dis 9, 150 (2018).

10. Huber-Lang, M. et al. Generation of C5a in the absence of C3: a new complement activation pathway. Nat Med 12, 682-7 (2006).

11. Bekassy, Z.D. et al. Aliskiren inhibits renin-mediated complement activation. Kidney Int 94, 689-700 (2018).

12. Krisinger, M.J. et al. Thrombin generates previously unidentified C5 products that support the terminal complement activation pathway. Blood 120, 1717-25 (2012).

13. Medler, T.R. et al. Complement C5a Fosters Squamous Carcinogenesis and Limits T Cell Response to Chemotherapy. Cancer Cell 34, 561-578 e6 (2018).

14. Nitta, H. et al. Cancer cells release anaphylatoxin $\mathrm{C} 5 \mathrm{a}$ from $\mathrm{C} 5$ by serine protease to enhance invasiveness. Oncol Rep 32, 1715-9 (2014).

15. West, E.E., Kolev, M. \& Kemper, C. Complement and the Regulation of T Cell Responses. Annu Rev Immunol 36, 309-338 (2018).

16. Hess, C. \& Kemper, C. Complement-Mediated Regulation of Metabolism and Basic Cellular Processes. Immunity 45, 240-54 (2016).

17. Arbore, G., Kemper, C. \& Kolev, M. Intracellular complement - the complosome - in immune cell regulation. Mol Immunol 89, 2-9 (2017).

18. Liszewski, M.K. et al. Intracellular complement activation sustains T cell homeostasis and mediates effector differentiation. Immunity 39, 1143-57 (2013).

19. Arbore, G. et al. T helper 1 immunity requires complement-driven NLRP3 inflammasome activity in CD4(+) T cells. Science 352, aad1210 (2016).

20. Markiewski, M.M. et al. Modulation of the antitumor immune response by complement. Nat Immunol 9, 1225-35 (2008).

21. Roumenina LT et al. Tumor cells highjack macrophage-produced complement C1q to promote tumor growth. Cancer Immunology Research in press (2019).

22. Kwak, J.W. et al. Complement Activation via a C3a Receptor Pathway Alters CD4(+) T Lymphocytes and Mediates Lung Cancer Progression. Cancer Res 78, 143-156 (2018).

23. Arshad, A. et al. Restoration of mannose-binding lectin complement activity is associated with improved outcome in patients with advanced pancreatic cancer treated with gemcitabine and intravenous omega-3 fish oil. JPEN J Parenter Enteral Nutr 38, 214-9 (2014).

24. Ytting, H., Jensenius, J.C., Christensen, I.J., Thiel, S. \& Nielsen, H.J. Increased activity of the mannan-binding lectin complement activation pathway in patients with colorectal cancer. Scand J Gastroenterol 39, 674-9 (2004).

25. Reddel, C.J., Tan, C.W. \& Chen, V.M. Thrombin Generation and Cancer: Contributors and Consequences. Cancers (Basel) 11 (2019).

26. Bonavita, E. et al. PTX3 is an extrinsic oncosuppressor regulating complement-dependent inflammation in cancer. Cell 160, 700-714 (2015).

27. Nabizadeh, J.A. et al. The Complement C3a Receptor Contributes to Melanoma Tumorigenesis by Inhibiting Neutrophil and CD4+ T Cell Responses. J Immunol 196, 4783-92 (2016).

28. Janelle, V. et al. Transient complement inhibition promotes a tumor-specific immune response through the implication of natural killer cells. Cancer Immunol Res 2, 200-6 (2014).

29. Wang, Y. et al. Autocrine Complement Inhibits IL10-Dependent T-cell-Mediated Antitumor Immunity to Promote Tumor Progression. Cancer Discov 6, 1022-35 (2016). 
30. Guglietta, S. et al. Coagulation induced by C3aR-dependent NETosis drives protumorigenic neutrophils during small intestinal tumorigenesis. Nat Commun 7, 11037 (2016).

31. Gunn, L. et al. Opposing roles for complement component C5a in tumor progression and the tumor microenvironment. J Immunol 189, 2985-94 (2012).

32. Schmidt, J., Klempp, C., Buchler, M.W. \& Marten, A. Release of iC3b from apoptotic tumor cells induces tolerance by binding to immature dendritic cells in vitro and in vivo. Cancer Immunol Immunother 55, 31-8 (2006).

33. Liu, C.F. et al. Complement Receptor 3 Has Negative Impact on Tumor Surveillance through Suppression of Natural Killer Cell Function. Front Immunol 8, 1602 (2017).

34. Fridman, W.H., Pages, F., Sautes-Fridman, C. \& Galon, J. The immune contexture in human tumours: impact on clinical outcome. Nat Rev Cancer 12, 298-306 (2012).

35. Hajishengallis, G., Reis, E.S., Mastellos, D.C., Ricklin, D. \& Lambris, J.D. Novel mechanisms and functions of complement. Nat Immunol 18, 1288-1298 (2017).

36. Ling, G.S. et al. C1q restrains autoimmunity and viral infection by regulating CD8(+) T cell metabolism. Science 360, 558-563 (2018).

37. Kremlitzka, M. et al. Interaction of Serum-Derived and Internalized C3 With DNA in Human B Cells-A Potential Involvement in Regulation of Gene Transcription. Front Immunol 10, 493 (2019).

38. Elvington, M., Liszewski, M.K., Bertram, P., Kulkarni, H.S. \& Atkinson, J.P. A C3(H2O) recycling pathway is a component of the intracellular complement system. J Clin Invest 127, 970-981 (2017).

39. King, B.C. et al. Complement Component C 3 Is Highly Expressed in Human Pancreatic Islets and Prevents beta Cell Death via ATG16L1 Interaction and Autophagy Regulation. Cell Metab 29, 202-210 e6 (2019).

40. Kurihara, R. et al. C5a promotes migration, proliferation, and vessel formation in endothelial cells. Inflamm Res 59, 659-66 (2010).

41. Corrales, L. et al. Anaphylatoxin C5a creates a favorable microenvironment for lung cancer progression. J Immunol 189, 4674-83 (2012).

42. Nunez-Cruz, S. et al. Genetic and pharmacologic inhibition of complement impairs endothelial cell function and ablates ovarian cancer neovascularization. Neoplasia 14, 9941004 (2012).

43. Bandini, S. et al. Early onset and enhanced growth of autochthonous mammary carcinomas in C3-deficient Her2/neu transgenic mice. Oncoimmunology 2, e26137 (2013).

44. Bulla, R. et al. C1q acts in the tumour microenvironment as a cancer-promoting factor independently of complement activation. Nat Commun 7, 10346 (2016).

45. Singh, J., Ahmed, A. \& Girardi, G. Role of complement component C1q in the onset of preeclampsia in mice. Hypertension 58, 716-24 (2011).

46. Bandini, $\mathrm{S}$. et al. The non-inflammatory role of $\mathrm{C} 1 \mathrm{q}$ during Her2/neu-driven mammary carcinogenesis. Oncoimmunology 5, e1253653 (2016).

47. Jourde-Chiche, N. et al. Endothelium structure and function in kidney health and disease. Nat Rev Nephrol 15, 87-108 (2019).

48. Roumenina, L.T., Rayes, J., Frimat, M. \& Fremeaux-Bacchi, V. Endothelial cells: source, barrier, and target of defensive mediators. Immunol Rev 274, 307-329 (2016).

49. Cho, M.S. et al. Autocrine effects of tumor-derived complement. Cell Rep 6, 1085-1095 (2014).

50. Doerner, S.K. et al. High-Fat Diet-Induced Complement Activation Mediates Intestinal Inflammation and Neoplasia, Independent of Obesity. Mol Cancer Res 14, 953-965 (2016).

51. Bouwens van der Vlis, T.A.M. et al. The complement system in glioblastoma multiforme. Acta Neuropathol Commun 6, 91 (2018).

52. Cho, M.S. et al. Complement Component 3 Is Regulated by TWIST1 and Mediates EpithelialMesenchymal Transition. J Immunol 196, 1412-8 (2016). 
53. Hu, W.H. et al. C5a receptor enhances hepatocellular carcinoma cell invasiveness via activating ERK1/2-mediated epithelial-mesenchymal transition. Exp Mol Pathol 100, 101-8 (2016).

54. Kaida, T. et al. C5a receptor (CD88) promotes motility and invasiveness of gastric cancer by activating RhoA. Oncotarget 7, 84798-84809 (2016).

55. Fan, Z., Qin, J., Wang, D. \& Geng, S. Complement C3a promotes proliferation, migration and stemness in cutaneous squamous cell carcinoma. J Cell Mol Med (2019).

56. Vlaicu, S.I. et al. Role of C5b-9 complement complex and response gene to complement-32 (RGC-32) in cancer. Immunol Res 56, 109-21 (2013).

57. Boire, A. et al. Complement Component 3 Adapts the Cerebrospinal Fluid for Leptomeningeal Metastasis. Cell 168, 1101-1113 e13 (2017).

58. Ajona, D., Ortiz-Espinosa, S., Pio, R. \& Lecanda, F. Complement in Metastasis: A Comp in the Camp. Front Immunol 10, 669 (2019).

59. Oostindie, S.C. et al. CD20 and CD37 antibodies synergize to activate complement by Fcmediated clustering. Haematologica (2019).

60. Riihila, P.M. et al. Complement factor $\mathrm{H}$ : a biomarker for progression of cutaneous squamous cell carcinoma. J Invest Dermatol 134, 498-506 (2014).

61. Riihila, P. et al. Complement factor I promotes progression of cutaneous squamous cell carcinoma. J Invest Dermatol 135, 579-588 (2015).

62. Sakai, N. et al. Tumorigenicity of BALB3T3 A31 cells transfected with hamster-complementC1s cDNA. Int J Cancer 58, 309-13 (1994).

63. Block, I. et al. CFP suppresses breast cancer cell growth by TES-mediated upregulation of the transcription factor DDIT3. Oncogene (2019).

64. Hong, Q. et al. Complement C1q activates tumor suppressor WWOX to induce apoptosis in prostate cancer cells. PLoS One 4, e5755 (2009).

65. Lubbers, R., van Essen, M.F., van Kooten, C. \& Trouw, L.A. Production of complement components by cells of the immune system. Clin Exp Immunol 188, 183-194 (2017).

66. Beatty, G.L. \& Gladney, W.L. Immune escape mechanisms as a guide for cancer immunotherapy. Clin Cancer Res 21, 687-92 (2015).

67. Mohme, M., Riethdorf, S. \& Pantel, K. Circulating and disseminated tumour cells mechanisms of immune surveillance and escape. Nat Rev Clin Oncol 14, 155-167 (2017).

68. Blok, V.T. et al. A possible role of CD46 for the protection in vivo of human renal tumor cells from complement-mediated damage. Lab Invest 80, 335-44 (2000).

69. Cancer Genome Atlas Research, N. et al. The Cancer Genome Atlas Pan-Cancer analysis project. Nat Genet 45, 1113-20 (2013).

70. Uhlen, M. et al. A pathology atlas of the human cancer transcriptome. Science 357 (2017).

71. Buettner, R. et al. Activated signal transducers and activators of transcription 3 signaling induces CD46 expression and protects human cancer cells from complement-dependent cytotoxicity. Mol Cancer Res 5, 823-32 (2007).

72. Ravindranath, N.M. \& Shuler, C. Expression of complement restriction factors (CD46, CD55 \& CD59) in head and neck squamous cell carcinomas. J Oral Pathol Med 35, 560-7 (2006).

73. Junnikkala, $\mathrm{S}$. et al. Secretion of soluble complement inhibitors factor $\mathrm{H}$ and factor $\mathrm{H}$-like protein (FHL-1) by ovarian tumour cells. Br J Cancer 87, 1119-27 (2002).

74. Surowiak, P. et al. CD46 expression is indicative of shorter revival-free survival for ovarian cancer patients. Anticancer Res 26, 4943-8 (2006).

75. Wilczek, E. et al. The possible role of factor $\mathrm{H}$ in colon cancer resistance to complement attack. Int J Cancer 122, 2030-7 (2008).

76. Ajona, D. et al. Expression of complement factor $\mathrm{H}$ by lung cancer cells: effects on the activation of the alternative pathway of complement. Cancer Res 64, 6310-8 (2004).

77. Olcina, M.M. et al. Mutations in an Innate Immunity Pathway Are Associated with Poor Overall Survival Outcomes and Hypoxic Signaling in Cancer. Cell Rep 25, 3721-3732 e6 (2018). 
78. Okroj, M., Corrales, L., Stokowska, A., Pio, R. \& Blom, A.M. Hypoxia increases susceptibility of non-small cell lung cancer cells to complement attack. Cancer Immunol Immunother 58, 1771-80 (2009).

79. Phillips, M.E., Rother, U. \& Rother, K. Serum complement in the rejection of sarcoma I ascites tumor grafts. J Immunol 100, 493-500 (1968).

80. Eilber, F.R. \& Morton, D.L. Demonstration in sarcoma patients of anti-tumor antibodies which fix only human complement. Nature 225, 1137-8 (1970).

81. Savola, S. et al. High Expression of Complement Component 5 (C5) at Tumor Site Associates with Superior Survival in Ewing's Sarcoma Family of Tumour Patients. ISRN Oncol 2011, 168712 (2011).

82. Liu, L., Li, W., Li, Z. \& Kirschfink, M. Sublytic complement protects prostate cancer cells from tumour necrosis factor-alpha-induced cell death. Clin Exp Immunol 169, 100-8 (2012).

83. Manning, M.L., Williams, S.A., Jelinek, C.A., Kostova, M.B. \& Denmeade, S.R. Proteolysis of complement factors iC $3 \mathrm{~b}$ and $\mathrm{C} 5$ by the serine protease prostate-specific antigen in prostatic fluid and seminal plasma. J Immunol 190, 2567-74 (2013).

84. Klikovits, T. et al. Circulating complement component $4 d(C 4 d)$ correlates with tumor volume, chemotherapeutic response and survival in patients with malignant pleural mesothelioma. Sci Rep 7, 16456 (2017).

85. Agostinis, C. et al. Complement Protein C1q Binds to Hyaluronic Acid in the Malignant Pleural Mesothelioma Microenvironment and Promotes Tumor Growth. Front Immunol 8, 1559 (2017).

86. Lucas, S.D. et al. Tumor-specific deposition of immunoglobulin $G$ and complement in papillary thyroid carcinoma. Hum Pathol 27, 1329-35 (1996).

87. Goslings, W.R. et al. Membrane-bound regulators of complement activation in uveal melanomas. CD46, CD55, and CD59 in uveal melanomas. Invest Ophthalmol Vis Sci 37, 188491 (1996).

88. Bacolod, M.D. et al. Immune infiltration, glioma stratification, and therapeutic implications. Trans/ Cancer Res 5, S652-S656 (2016).

89. Bouwens, T.A. et al. Complement activation in Glioblastoma multiforme pathophysiology: evidence from serum levels and presence of complement activation products in tumor tissue. J Neuroimmunol 278, 271-6 (2015).

90. Ajona, D. et al. Investigation of complement activation product c4d as a diagnostic and prognostic biomarker for lung cancer. J Natl Cancer Inst 105, 1385-93 (2013).

91. Germain, C. et al. Presence of B cells in tertiary lymphoid structures is associated with a protective immunity in patients with lung cancer. Am J Respir Crit Care Med 189, 832-44 (2014).

92. Montfort, A. et al. A Strong B-cell Response Is Part of the Immune Landscape in Human HighGrade Serous Ovarian Metastases. Clin Cancer Res 23, 250-262 (2017).

93. Kroeger, D.R., Milne, K. \& Nelson, B.H. Tumor-Infiltrating Plasma Cells Are Associated with Tertiary Lymphoid Structures, Cytolytic T-Cell Responses, and Superior Prognosis in Ovarian Cancer. Clin Cancer Res 22, 3005-15 (2016).

94. Yeong, J. et al. High Densities of Tumor-Associated Plasma Cells Predict Improved Prognosis in Triple Negative Breast Cancer. Front Immunol 9, 1209 (2018).

95. Ajona, D. et al. Complement C4d-specific antibodies for the diagnosis of lung cancer. Oncotarget 9, 6346-6355 (2018).

96. Xi, W. et al. High Level of Anaphylatoxin C5a Predicts Poor Clinical Outcome in Patients with Clear Cell Renal Cell Carcinoma. Sci Rep 6, 29177 (2016).

97. $\mathrm{Xi}, \mathrm{W}$. et al. Enrichment of $\mathrm{C} 5 \mathrm{a}-\mathrm{C} 5 \mathrm{aR}$ axis predicts poor postoperative prognosis of patients with clear cell renal cell carcinoma. Oncotarget 7, 80925-80934 (2016).

98. Capello, M. et al. Exosomes harbor B cell targets in pancreatic adenocarcinoma and exert decoy function against complement-mediated cytotoxicity. Nat Commun 10, 254 (2019). 
99. Chen, J. et al. Expression and clinical significance of complement C3, complement C4b1 and apolipoprotein E in pancreatic cancer. Oncol Lett 6, 43-48 (2013).

100. Ginhoux, F. \& Guilliams, M. Tissue-Resident Macrophage Ontogeny and Homeostasis. Immunity 44, 439-449 (2016).

101. Komohara, Y. et al. Macrophage infiltration and its prognostic relevance in clear cell renal cell carcinoma. Cancer Sci 102, 1424-31 (2011).

102. Mantovani, A., Sozzani, S., Locati, M., Allavena, P. \& Sica, A. Macrophage polarization: tumorassociated macrophages as a paradigm for polarized M2 mononuclear phagocytes. Trends Immunol 23, 549-55 (2002).

103. Chevrier, S. et al. An Immune Atlas of Clear Cell Renal Cell Carcinoma. Cell 169, 736-749 e18 (2017).

104. Clarke, E.V., Weist, B.M., Walsh, C.M. \& Tenner, A.J. Complement protein C1q bound to apoptotic cells suppresses human macrophage and dendritic cell-mediated Th17 and Th1 T cell subset proliferation. J Leukoc Biol 97, 147-60 (2015).

105. Ghebrehiwet, B. et al. Evidence that the two C1q binding membrane proteins, gC1q-R and cC1q-R, associate to form a complex. J Immunol 159, 1429-36 (1997).

106. Imamura, T. et al. Influence of the $\mathrm{C} 5 \mathrm{a}-\mathrm{C} 5$ a receptor system on breast cancer progression and patient prognosis. Breast Cancer 23, 876-885 (2016).

107. Chang, I.W. et al. Complement Component 1, s Subcomponent Overexpression is an Independent Poor Prognostic Indicator in Patients with Urothelial Carcinomas of the Upper Urinary Tract and Urinary Bladder. J Cancer 7, 1396-405 (2016).

108. Nasr, S.H., Fidler, M.E. \& Said, S.M. Paraffin Immunofluorescence: A Valuable Ancillary Technique in Renal Pathology. Kidney Int Rep 3, 1260-1266 (2018).

109. Zha, H. et al. Intracellular Activation of Complement C3 Leads to PD-L1 Antibody Treatment Resistance by Modulating Tumor-Associated Macrophages. Cancer Immunol Res 7, 193-207 (2019).

110. Ajona, D. et al. A Combined PD-1/C5a Blockade Synergistically Protects against Lung Cancer Growth and Metastasis. Cancer Discov 7, 694-703 (2017).

111. Ricklin, D., Mastellos, D.C., Reis, E.S. \& Lambris, J.D. The renaissance of complement therapeutics. Nat Rev Nephrol 14, 26-47 (2018).

112. Pio, R., Ajona, D., Ortiz-Espinosa, S., Mantovani, A. \& Lambris, J.D. Complementing the Cancer-Immunity Cycle. Frontiers in Immunology 10 (2019).

113. Surace, L. et al. Complement is a central mediator of radiotherapy-induced tumor-specific immunity and clinical response. Immunity 42, 767-77 (2015).

114. Facciabene, A. et al. Local endothelial complement activation reverses endothelial quiescence, enabling t-cell homing, and tumor control during t-cell immunotherapy. Oncoimmunology 6, e1326442 (2017).

115. Diebolder, C.A. et al. Complement is activated by IgG hexamers assembled at the cell surface. Science 343, 1260-3 (2014).

116. de Jong, R.N. et al. A Novel Platform for the Potentiation of Therapeutic Antibodies Based on Antigen-Dependent Formation of IgG Hexamers at the Cell Surface. PLoS Biol 14, e1002344 (2016).

117. Lee, C.H. et al. IgG Fc domains that bind C1q but not effector Fcgamma receptors delineate the importance of complement-mediated effector functions. Nat Immunol 18, 889-898 (2017).

118. Fishelson, Z. \& Kirschfink, M. Complement C5b-9 and Cancer: Mechanisms of Cell Damage, Cancer Counteractions, and Approaches for Intervention. Front Immunol 10, 752 (2019).

119. Hoadley, K.A. et al. Cell-of-Origin Patterns Dominate the Molecular Classification of 10,000 Tumors from 33 Types of Cancer. Cell 173, 291-304 e6 (2018).

120. Cerami, E. et al. The cBio cancer genomics portal: an open platform for exploring multidimensional cancer genomics data. Cancer Discov 2, 401-4 (2012). 
121. Tang, Z. et al. GEPIA: a web server for cancer and normal gene expression profiling and interactive analyses. Nucleic Acids Res 45, W98-W102 (2017).

\section{Figure legends}

Figure 1. The complement system in the TME. A) The mechanisms of activation and regulation of the complement system. The complement system can be activated by three pathways; the classical, lectin and alternative pathway leading to the generation of the C3 convertase. The classical pathway is activated after the recognition by $\mathrm{C} 1$ complex (composed of $\mathrm{C} 1 \mathrm{q}, \mathrm{C} 1 \mathrm{r}$ and $\mathrm{C} 1 \mathrm{~s}$ ) of immune complexes/apoptotic cells; the lectin pathway is initiated after the fixation of the complex MBL (Mannose Binding Lectin)/MASP (MBLassociated serine proteases) to terminal mannose residues. The alternative pathway is constitutively activated at low grade by the spontaneous hydrolysis of $\mathrm{C} 3$ into $\mathrm{C} 3 \mathrm{H} 2 \mathrm{O}$. These initiation events lead to the formation of enzymatic complexes - C3 convertases, which cleave the central component C3 into C3a (anaphylatoxin) and C3b which can opsonize the cells and allows the formation of the C5 convertase. The C5 convertase cleaves C5 into C5a (anaphylatoxin) and C5b which initiate the terminal pathway of the complement system, leading to the formation of the membrane attack complex. Some proteases, extrinsic to the cascade, can also cleave C3 or C5 independently to the convertases formation. To avoid host tissue damage, this system is tightly regulated by soluble or membranous proteins at different levels of the cascade.

B) The composition of the TME and the complement proteins, produced by different nonmalignant cell types. The tumor has a rich complement environment. All stromal and tumor cells participate to the local production of complement proteins. The immune cells, especially the myeloid ones, can produce components especially of the classical and alternative pathways, express high level of complement receptors and multiple regulators. The endothelial cells and fibroblasts are also key actors the TME and produce complement proteins, express regulators and a lower level of complement receptors. Finally, the participation of tumor cells depends of cancer type but a key feature is the high expression of complement regulators to protect against complement-dependent cytotoxicity. 
Figure 2. Modulation by complement of the pro- and anti-tumoral impacts of the immune contexture in cancers. A) pro-tumoral action of complement effectors on the immune cells; B) anti-tumoral action of complement effectors on the immune cells

Figure 3. The pro- and anti-tumoral impact of complement on neoangiogenesis and on the fundamental processes of tumor cell. The complement system can A) promote angiogenesis through the actions of anaphylatoxines of $\mathrm{C} 1 \mathrm{q}$ or can act on the key characteristics of tumor cells and B) induce a pro-tumoral phenotype by increasing EMT, autophagy, proliferation, migration and stemness or C) have a anti-tumoral impact by decreasing proliferation and apoptosis. These effects are cancer type dependent and concentration dependent.

Figure 4. Expression of complement genes in human cancers. TCGA PanCanAtlas ${ }^{119}$ data used are downloaded through cBioPortal $^{120}$ and come from TCGA Data Coordination Center (DCC). RNASeq expression data, RNASeqV2, from TCGA is processed and normalized using RSEM to generate TPM (transcripts per million). Specifically, the data_RNA_Seq_v2_expression_median file in cBioPortal corresponds to the rsem.genes.normalized_results file from TCGA. 30 solid tumor types are used in this analysis. In order to avoid bias, liver hepatocellular carcinoma (LIHC) was excluded from the study because of the capacity of the liver to express very high amounts of complement genes. The mean of TPM of each complement gene for the patients of the different TCGA cohorts is calculated and then converted in log2(1+TPM) data. Using R package "pheatmap" and the clustering method "complete", the heatmap is generated and allows the visualization of the TPM mean of each complement gene for the different TCGA cohorts. ACC, adenocortical carcinoma; BLCA, bladder carcinoma; BRCA, breast carcinoma; CESC, cervical squamous carcinoma; $\mathrm{CHOL}$, cholangiocarcinoma; COAD, colon adenocarcinoma; DLBC, diffuse large B-cell lymphoma; ESCA, esophageal carcinoma; GBM, glioblastoma multiforme; HNSC, head and neck carcinoma; $\mathrm{KICH}$, kidney chromophobe; KIRC, kidney renal clear cell carcinoma; KIRP, kidney renal papillary cell carcinoma; LGG, lower grade glioma; LUAD, lung adenocarcinoma; LUSC, lung squamous carcinoma; MESO, mesothelioma; OV, ovarian serous cystadenocarcinoma; PAAD, pancreatic adenocarcinoma; PRAD, prostate adenocarcinoma; READ, rectum adenocarcinoma; SARC, sarcoma; SKCM, skin cutaneous melanoma; STAD, stomach adenocarcinoma; TGCT, testicular germ cell tumors; THCA, thyroid carcinoma; THYM, thymoma; UCEC, uterine corpus endometrial carcinoma; UCS, uterine carcinosarcoma; UVM, uveal melanoma.

Figure 5. Impact of the expression of the complement genes on the survival of cancer patients. The survival analysis is performed by using Gene Expression Profiling Interactive Analysis (GEPIA) tool ${ }^{121}$. Overall survival (OS) analysis based on gene expression with a median cut-off is used to calculate hazards ratio based on Cox PH Model and Log-rank pvalue. Considering the very low number of events, TGCT cohort is excluded from the survival 
analysis. The heatmap representing the log2 HR is generated by using the $\mathrm{R}$ package "pheatmap" and the clustering method "ward.D2". The heatmap allows the visualization of the log $2 \mathrm{HR}$ with a scale centered in 0 of each complement gene for the different TCGA cohorts. The surrounded boxes correspond to a log-rank p-value $<0.05$.

Figure 6. Proposed mechanisms for the classical complement pathway activation and its consequences on tumor progression in tumors with "aggressive complement". C1q is produced by TAM (1) and contributes to a tumor-promoting phenotype of these cells (2) and T cell exhaustion. Secreted C1q promotes adherence of tumor cells ( 3 ) and neoangiogenesis (4). A particular feature of ccRCC is that the tumor cells produce C1r and C1s (5) and allow formation of a functionally active $\mathrm{C} 1$ complex (6), capable to activate the classical pathway. Moreover, IgG deposits on tumor cells serve as C1 ligand (7) to initiate the cascade. The tumor cells produce also the subsequent components and allow C4 and C3 activation fragments deposition (8). Anaphylatoxins $\mathrm{C} 3 \mathrm{a}$ and $\mathrm{C} 5 \mathrm{a}$ are released, exerting their action on the tumor cells and on their microenvironment. The ensemble of these processes contributes to tumor progression and poor prognosis of patients. This model is based on the data for KIRC and is potentially applicable in other tumors from this group. 
Figure 1A






\section{Figure 1B}



$C 3, F B, F D, F P$
$C 5, C 7$

\section{MASP-1}

$\mathrm{C} 1 \mathrm{q}, \mathrm{C} 1 \mathrm{r}, \mathrm{C} 1 \mathrm{~s}, \mathrm{C} 4, \mathrm{C} 2$



\begin{tabular}{|ll|}
\hline 1 & Regulators \\
$\mathbb{1}$ & C3AR \\
C5aR \\
C3a \\
C5a
\end{tabular}




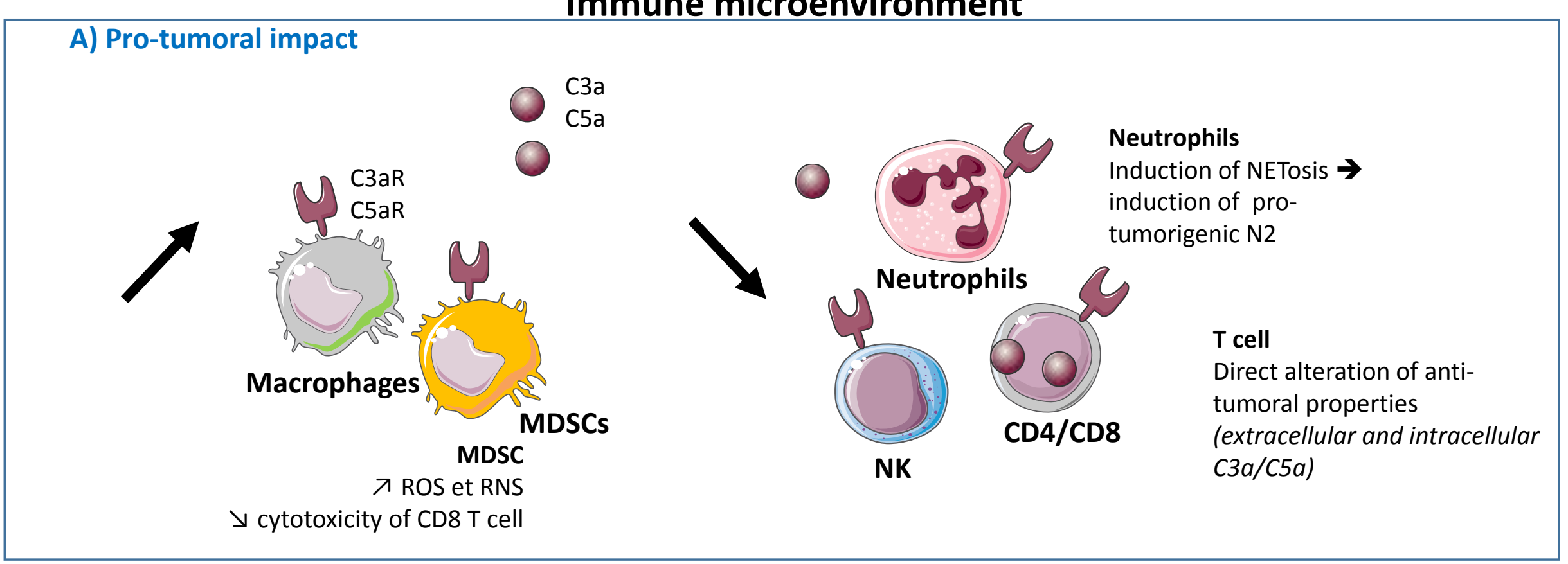

B) Anti-tumoral impact


$\Rightarrow \quad \lambda$ recruitment of NK and M1 macrophages

NK



$7 \mathrm{CD} 4$ and $\mathrm{CD} 8 \mathrm{~T}$ cells :

$\Longrightarrow$ recrutement and cytotoxic effect

$\lambda$ IFN-y production 


\section{Angiogenesis}



\section{Tumor cells}

Figure 5








